\title{
Staged hybrid approach for an acute-on-chronic aortic dissection with rupture in a Jehovah's Witness patient: Case report
}

\author{
Sarah T. Ward, MD, ${ }^{\mathrm{a}}$ Minha \\ Ann Arbor, Mich \\ From the a Department of Cardiac Surgery, University of Michigan Frankel Cardiovascular Center, Ann Arbor, \\ Mich; and the ${ }^{\mathrm{b}}$ Department of Radiology, University of Michigan Frankel Cardiovascular Center, Ann Arbor, \\ Mich. \\ Disclosures: David Williams reports consulting fees from Boston Scientific and Gore and Associates. All other \\ authors have nothing to disclose with regard to commercial support. \\ Received for publication May 22, 2015; revisions received July 24, 2015; accepted for publication July 24, 2015; \\ available ahead of print Aug 22, 2015 \\ Address for reprints: Bo Yang, MD, PhD, Department of Cardiac Surgery, Cardiovascular Center, The University \\ of Michigan, Ann Arbor, MI 48109 (E-mail: boya@med.umich.edu). \\ J Thorac Cardiovasc Surg 2015;150:e63-5 \\ $0022-5223 / \$ 36.00$ \\ Copyright (C) 2015 by The American Association for Thoracic Surgery \\ http://dx.doi.org/10.1016/j.jtcvs.2015.07.080
}

We present a first case report of a Jehovah's Witness patient with an acute-on-chronic type $\mathrm{B}$ aortic dissection that resulted in aortic rupture and right hemothorax and was successfully managed with an initial thoracic endovascular aortic repair (TEVAR) followed by open repair.

\section{CLINICAL SUMMARY}

The patient was a 69-year-old man with a history of an acute type A aortic dissection repair in 1999. In 2012, follow-up computed tomographic imaging revealed a 7.0-cm descending thoracic aortic aneurysm (dTAA; Figure 1, A). Because the patient was a Jehovah's Witness, he did not pursue surgery in light of the high operative risk of mortality without transfusion of blood products. In 2014, the patient had a rupture of his dTAA, resulting in right hemothorax and acute hemorrhagic shock (hemoglobin concentration of $9 \mathrm{~g} / \mathrm{dL}$ ). He was referred to hospice care at an outside hospital. Careful review of computed tomographic imaging revealed a new connection between the true lumen and a new false lumen, which was ruptured, representing an acute-on-chronic type $\mathrm{B}$ aortic dissection, a dTAA of $8.0 \mathrm{~cm}$ (Figure 1, $B$ and $C$ ), and an aortic arch aneurysm of 4.5 to $5 \mathrm{~cm}$.

The patient was urgently transferred to the University of Michigan Health System, and a 2-stage repair was performed. In stage 1 , the bleeding of the ruptured dTAA was stopped by placement of a C-TAG Gore 30$\mathrm{mm} \times 10-\mathrm{cm}$ stent graft in the true lumen to cover the acute tear between the true lumen and the new false lumen, which was sized by the proximal true lumen (Figure $1, D$ ). Subsequently, the patient was treated with a subcutaneous injection of 20,000 units of epoetin alfa (Epogen) every other day, as well as vitamin $\mathrm{C}$ and intravenous iron daily, for a course of 8 weeks. The hemoglobin level recovered from $9.1 \mathrm{~g} / \mathrm{dL}$ to $16.9 \mathrm{~g} / \mathrm{dL}$. In stage 2 , open repair of the dTAA from the distal arch to T11 was performed through 4th was $12.6 \mathrm{~g} / \mathrm{dL}$.

\section{DISCUSSION}

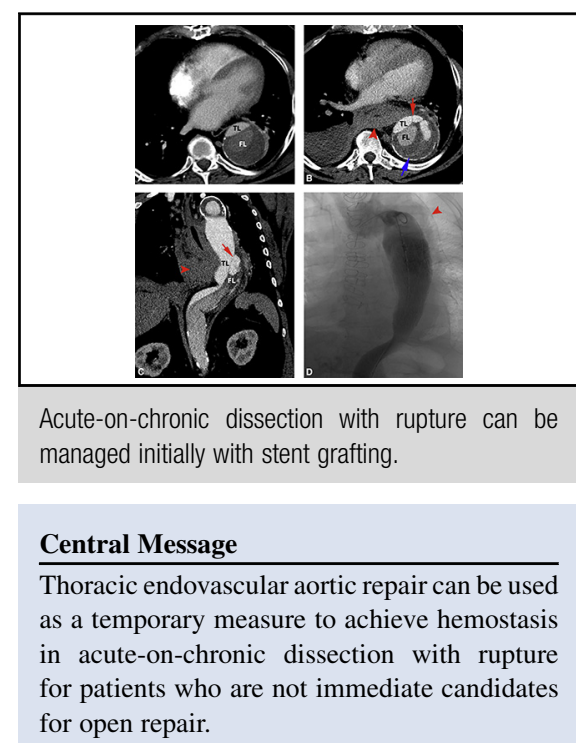

See Editorial Commentary page e67.

and 8th thoracotomies (Figure 2, $A$ and $B$ ). The aortic arch was clamped between the left common carotid artery and left subclavian artery for the proximal anastomosis. Distally, the aorta was divided obliquely from the T11 to the T10 level to preserve the T10 intercostal arteries. For both operations (stages 1 and 2), lumbar drains were placed for spinal protection. The cardiopulmonary bypass time was 2 hours and 12 minutes; the distal aortic crossclamp time was 1 hour and 36 minutes. The patient had excellent hemostasis after protamine and only received autologous blood. His immediate postoperative hemoglobin concentration

Acute-on-chronic dissection with aortic rupture is rare. ${ }^{1}$ Traditional wisdom calls for urgent or emergency open repair of the acute redissected aorta. Immediate open repair was not an option, however, because this patient was a Jehovah's Witness who had anemia and was in hemorrhagic shock. To our knowledge, this is the first case report to use the staged approach described here, including TEVAR followed open repair, for this presentation.

The first challenge in this case was to make an initial diagnosis of acute-on-chronic dissection and decide where to place the stent graft to achieve hemostasis 

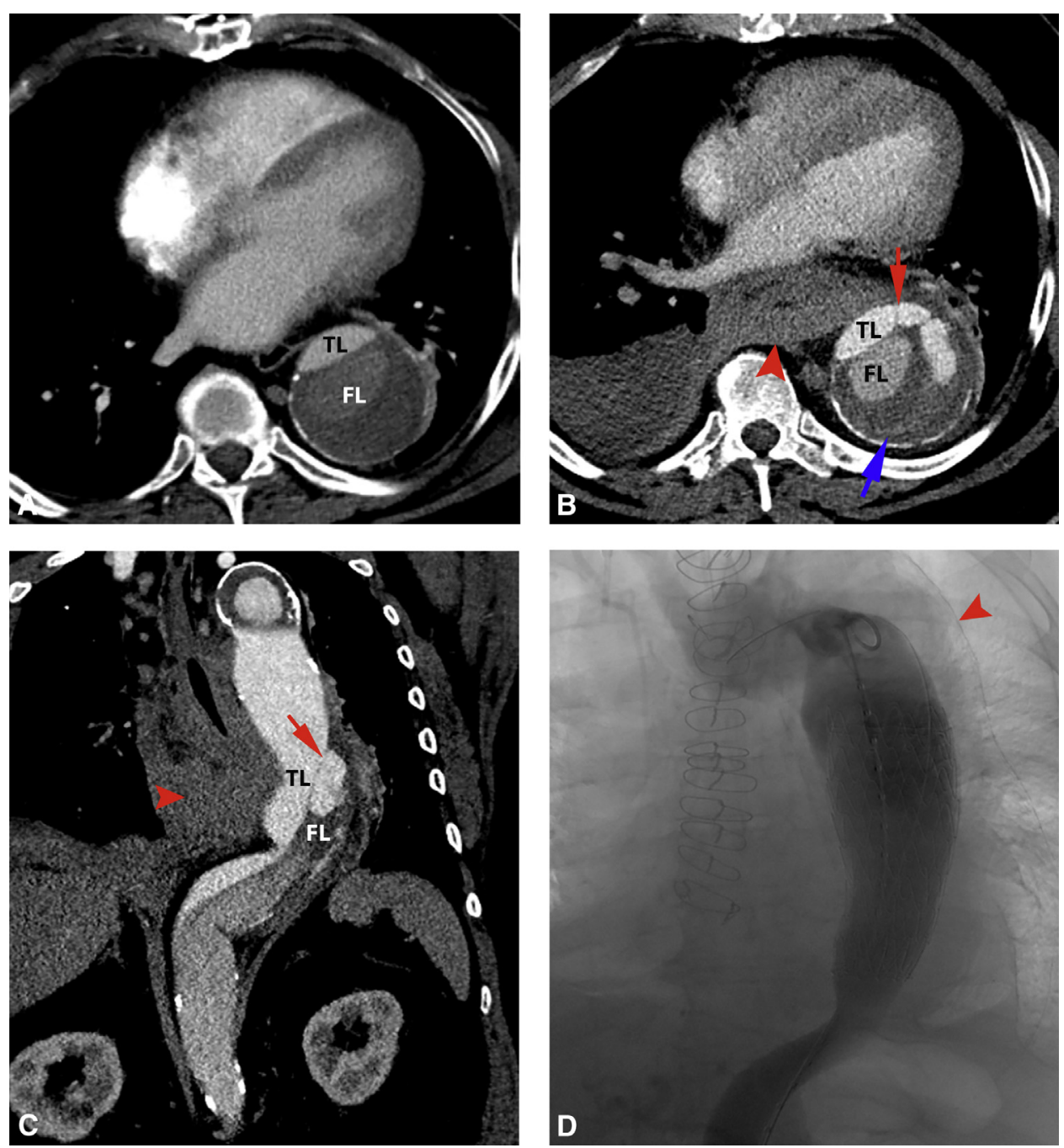

FIGURE 1. Stage 1 operation. A, An axial image from a computed tomographic angiogram 3 years before the aortic rupture demonstrates the previous appearance the of true lumen $(T L)$ and false lumen $(F L)$, with thrombus within much of the false lumen. B, By way of a new intimal tear, contrast material passes from the true lumen through a new false lumen channel (red arrow) in the thrombus of the chronic false lumen and merges with the chronic false lumen at the level of the diaphragm (not shown). Also notable is hyperdensity within the false lumen (blue arrow), which was present on unenhanced images (not shown) representing acute blood. Mediastinal hematoma (red arrowhead) and the true lumen are also visible. C, The coronal reconstruction of computed tomographic angiography at the same level as in panel $A$ shows the new intimal tear (red arrow), the true lumen, the chronic false lumen, and the mediastinal hematoma (red arrowhead). D, Thoracic aortography after endograft placement confirmed patency of the endograft without flow across the new intimal tear. Also notable is calcification along the false lumen (red arrowhead).

because there were 3 lumens. It was critical to recognize the mechanism of the bleeding from the true lumen into the new false lumen and design the stent graft to cover the new primary tear in the true lumen and achieve hemostasis.

The second challenge was how to definitively repair the aortic arch aneurysm $(4.5-5.0 \mathrm{~cm})$ and dTAA. One consideration was a first-stage resternotomy with total arch replacement and elephant trunk followed by a second-stage open repair of the dTAA. In this clinical scenario, however, the dTAA was ruptured and life-threatening, and it therefore was repaired first. The dilation of the arch made the crossclamping of the arch difficult. We had to pull an umbilical tape surrounding the aortic arch against the crossclamp to stop the bleeding completely between the crossclamp for the proximal anastomosis.

The third challenge was urgent open repair of the dTAA in this Jehovah's Witness patient who was unwilling to accept blood transfusion. Preoperative treatment with highdose epoetin alfa, iron, and vitamin C successfully increased the patient's hemoglobin concentration. ${ }^{2}$ We also chose crossclamping of the aortic arch for the proximal anastomosis instead of hypothermic circulatory arrest to avoid prolonged 

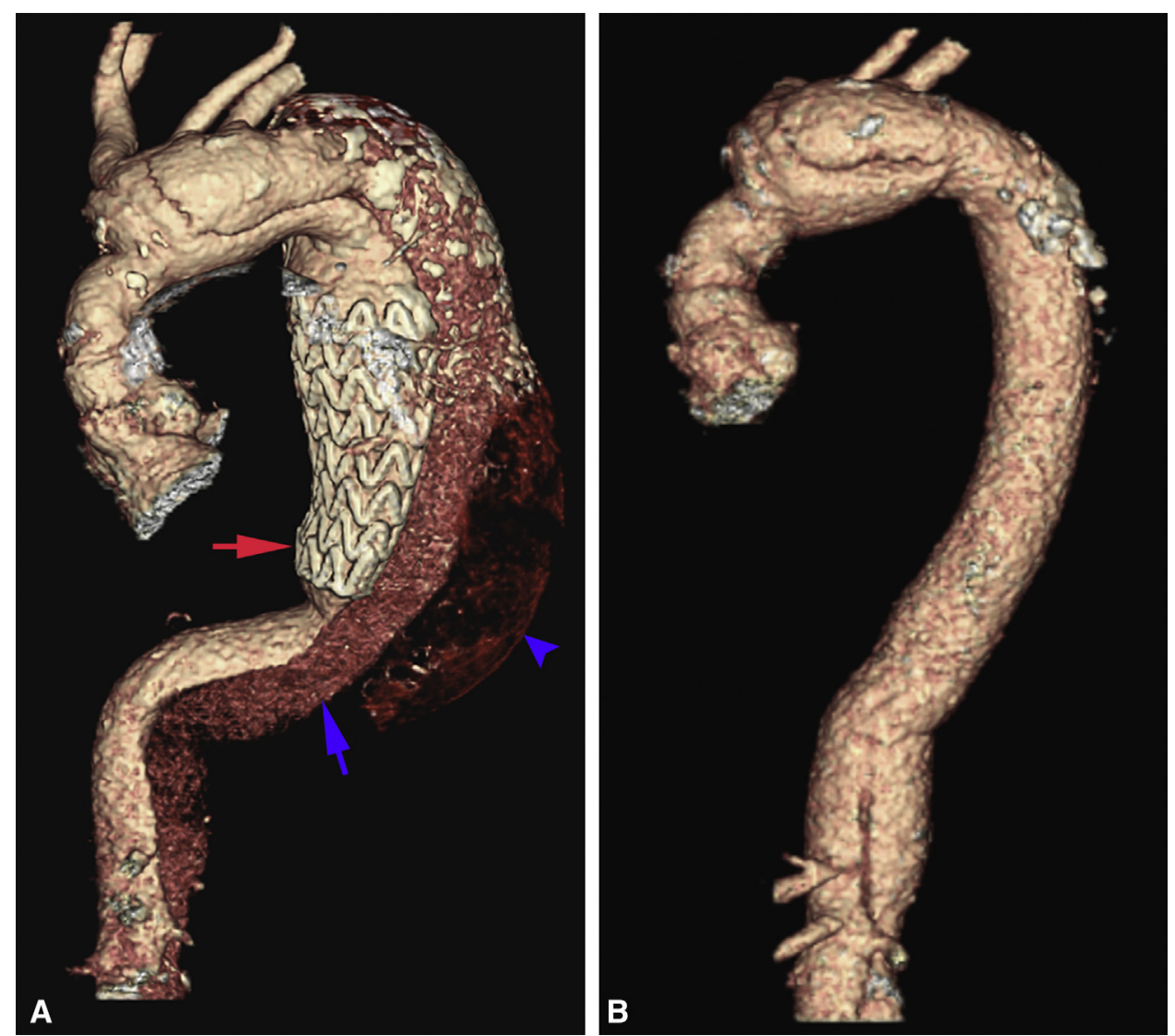

FIGURE 2. Stage 2 operation. Three-dimensional reconstruction of the thoracic aorta from computed tomographic angiography before (A) and after (B) open repair. A, The endograft can be seen within the true lumen (red arrow), flow within the false lumen (blue arrow), and thrombus within the false lumen (blue arrowhead). B, The endograft was explanted and replaced with a 30-mm Hemashield vascular graft.

cardiopulmonary bypass time and coagulopathy. We obtained 4 units of autologous blood before cardiopulmonary bypass, which helped the hemostasis significantly.

In summary, we report a novel approach (staged TEVAR and open repair) for a rare problem (acute-on-chronic aortic dissection with rupture) in an uncommon situation (a Jehovah's Witness patient who declined blood transfusion). TEVAR can be used as a temporary measure to achieve hemostasis in acute-on-chronic aortic dissection with rupture for patients who are not immediate candidates for open repair.

\section{References}

1. Abe H, Funaki S, Chiba K, Kitanaka Y, Makuuchi H. Traumatic rupture of the false lumen in a patient with preexisting chronic dissection of the descending thoracic aorta. Gen Thorac Cardiovasc Surg. 2011;59:559-62.

2. Jassar AS, Ford PA, Haber HL, Isidro A, Swain JD, Bavaria JE, et al. Cardiac surgery in Jehovah's Witness patients: ten-year experience. Ann Thorac Surg. 2012; 93:19-25. 